\title{
Size-induced superantiferromagnetism with reentrant spin-glass behavior in metallic nanoparticles of $\mathrm{TbCu}_{2}$
}

\author{
C. Echevarria-Bonet, ${ }^{1}$ D. P. Rojas, ${ }^{2}$ J. I. Espeso, ${ }^{1}$ J. Rodríguez Fernández, ${ }^{1}$ L. Rodríguez Fernández, ${ }^{3}$ P. Gorria, \\ J. A. Blanco, ${ }^{4}$ M. L. Fdez-Gubieda, ${ }^{5}$ E. Bauer, ${ }^{6}$ G. André, ${ }^{7}$ and L. Fernández Barquín ${ }^{1}$ \\ ${ }^{1}$ Departamento CITIMAC, Universidad de Cantabria, 39005 Santander, Spain \\ ${ }^{2}$ Departamento de Física e Instalaciones Aplicadas-ETSAM, 28040 Madrid, Spain \\ ${ }^{3}$ SERMET, Universidad de Cantabria, 39005 Santander, Spain \\ ${ }^{4}$ Departamento Física, Universidad de Oviedo, 33007 Oviedo, Spain \\ ${ }^{5}$ Depto. Electricidad y Electrónica, Universidad del País Vasco (UPV/EHU), 48940 Leioa and BCMaterials, \\ Building No. 500, Technological Park of Biscay, 48160 Derio, Spain \\ ${ }^{6}$ Institut für Festkörperphysik, Technische Universität Wien, 1040 Wien, Austria \\ ${ }^{7}$ Laboratoire Léon Brillouin, CEA-SACLAY, 91191 Gif-sur-Yvette Cedex, France
}

(Received 24 January 2013; revised manuscript received 3 May 2013; published 24 May 2013)

\begin{abstract}
An unusual $4 f$-superantiferromagnetic state characterized by simultaneous antiferromagnetic and spin-glass behaviors induced by particle-size reduction is revealed in metallic nanoparticles $(\approx 9 \mathrm{~nm})$ of $\mathrm{TbCu}_{2}$. The Néel temperature is $46 \mathrm{~K}$ and the glassy freezing is below $\approx 9 \mathrm{~K}$ and governed by a critical slowing down process. Neutron diffraction at $1.8 \mathrm{~K}$ establishes the superantiferromagnetism. The latter is settled by the nanoparticle moments and the freezing mechanism is provided by the surface spins.
\end{abstract}

DOI: 10.1103/PhysRevB.87.180407

PACS number(s): 75.10.Nr, 75.25.-j, 75.50.Lk, 75.75.-c

The family tree of magnetism is formed by several archetypal arrangements, which are manifestations of the more general physical order/disorder behavior found in various systems such as liquid crystals and superconductors. Indeed, antiferromagnetism (AF) and the glassy spin (spin glass, SG) state have been thoroughly described in numerous materials. ${ }^{1-3}$ Other branches of such a tree are based on mictomagnetism (cluster glass) and superparamagnetism, the latter associated with magnetic nanoparticles. ${ }^{1-5}$ The advent of new production techniques and research in new materials have spawned a variety of behaviors, consequences of competing magnetic interactions. In this sense, ferromagnets with reentrant state, $, 2,4,6$ superferromagnets, or superspin glasses ${ }^{7-9}$ display new spin dispositions derived from the magnetic interactions.

The coexistence of ferromagnetism (FM) and spin glass has been evidenced through the Curie transition and a further SG freezing at lower temperatures. This finding could be observed in either crystalline ${ }^{1,4}$ or amorphous materials. ${ }^{6,10}$ Surprisingly, that coexistence has been sparsely reported for the AF-SG arrangements. ${ }^{1-15}$ Reports on Ising $\mathrm{Fe}_{0.55} \mathrm{Mg}_{0.45} \mathrm{Cl}_{2}$ and $\mathrm{Fe}_{0.6} \mathrm{Mn}_{0.4} \mathrm{TiO}_{3}{ }^{11}$ compounds, $\mathrm{YBa}_{2}\left(\mathrm{Cu}_{0.88} \mathrm{Fe}_{0.12}\right)_{3} \mathrm{O}_{6.5}$ semiconductors, ${ }^{12}$ insulating materials of $\mathrm{Co}_{2}(\mathrm{OH})\left(\mathrm{PO}_{4}\right),{ }^{13}$ and Heisenberg perovskites of $\mathrm{Pb}\left(\mathrm{Fe}_{1 / 2} \mathrm{Nb}_{1 / 2}\right) \mathrm{O}_{3}{ }^{14,15}$ have been delivered. The phenomenal research output from magnetic nanoparticles ignited the idea of producing such AFSG coexistence by a structure comprising a collection of AF nanoparticles. The AF nanoparticles present a weak ferromagnetic component due to uncompensated spins and have been studied in the form of (pseudo)oxides. ${ }^{7,16}$ This gives rise to what Néel coined as superantiferromagnetism of individual particles. ${ }^{17}$ In nanometric $\mathrm{TbCu}_{2}$, one counts with the advantage of a high magnetic Tb signal, a Néel transition $\left(T_{N}\right)$ below room temperature, and the possibility to produce them in large quantities (suitable for neutron diffraction) via high-energy milling. ${ }^{18}$ In bulk state, $\mathrm{TbCu}_{2}$ crystallizes in the Imma symmetry (orthorhombic, $\mathrm{CeCu}_{2}$-type crystal structure) and orders at $T_{N}=48 \mathrm{~K}^{19}$ with a collinear $\mathrm{AF}$ structure. ${ }^{20}$ Seizing the particle size $(D)$ as the driving variable to induce spin rearrangements, we report here the existence of a macroscopic AF with a reentrant $\mathrm{SG}$ state in nanosized $\mathrm{TbCu}_{2}$ alloys.

A large $\left(\simeq 5\right.$ g) quantity of nanometric $\mathrm{TbCu}_{2}$ was produced by high-energy mechanical (WC containers) milling in $\mathrm{Ar}$ atmosphere $\left(99.99 \%\right.$ ) during 2 hours. $^{21}$ The master alloy was annealed at $750{ }^{\circ} \mathrm{C}$ for 5 days to ensure homogeneity prior to milling. In Fig. 1, the X-ray diffraction pattern performed at $\mathrm{RT}(\mathrm{Cu}-\mathrm{K} \alpha \lambda=1.5418 \AA)$ is shown. The pattern has been refined by the Rietveld method (FULLPROF ${ }^{22}$ ) including the resolution function with $\mathrm{LaB}_{6}$ calibration. The fit was carried out with a Thompson-Cox-Hastings function, allowing the calculation of the particle size $(D)$ and strain $(\epsilon)$. The result indicates that the Imma symmetry of the bulk alloy is retained and that the lattice parameters $a=4.322(1) \AA, b=6.833(2) \AA$, and $c=7.327(2) \AA$ are similar to those of the bulk alloy. ${ }^{19}$ The Bragg error $R_{B}=14.6 \%$ is low for a nanostructure. The size $D=9(1) \mathrm{nm}$ and $\epsilon=0.2(1) \%$. Thus, the grinding time to obtain nanoparticles is small compared to other milled RE intermetallics. ${ }^{21}$ The structural characterization was completed via transmission electron microscopy (TEM). The inset of Fig. 1 shows a bright-field image of $\mathrm{TbCu}_{2}$ nanoparticles with a sharp surface and absence of amorphous environments, and the high-resolution detail in which lattice planes are visible confirms the particle crystallinity. A histogram analysis gives $D_{\text {TEM }}=7(1) \mathrm{nm}$.

The static magnetic characterization $M_{\mathrm{DC}}(T)$ was recorded in zero-field cooled (ZFC) and field cooled (FC) curves, and the loops $M_{\mathrm{DC}}(H)(T=5 \mathrm{~K})$, including the initial magnetization at $T=5$ and $150 \mathrm{~K}$. In Fig. $2(H=1 \mathrm{kOe})$, the first manifestation of the $\mathrm{PM} \leftrightarrow \mathrm{AF} \leftrightarrow \mathrm{SG}$ transitions is patent. Thus, there is a peak at $T_{N}=46.8(5) \mathrm{K}$, followed by an increase of the susceptibility reaching another peak at a lower temperature, marking the freezing transition $T_{f}=9.2(2) \mathrm{K}$. 


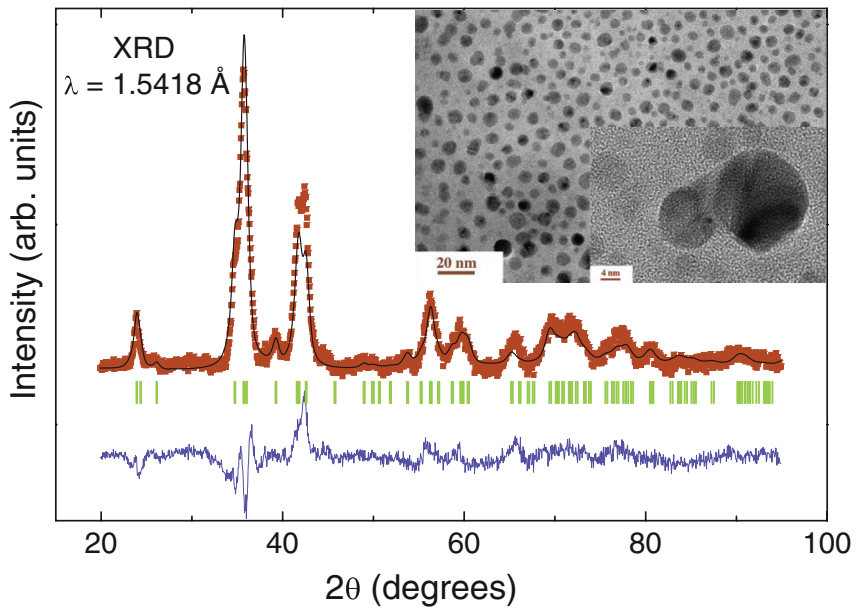

FIG. 1. (Color online) Rietveld refinement of the XRD pattern of $2 \mathrm{~h}$ milled alloy $(T=300 \mathrm{~K})$. The theoretical calculation is depicted as a continuous line and the experimental data as points. The blue line below the spectra is the difference between the calculated and experimental patterns. The size is $D=9(1) \mathrm{nm}$. Inset: Bright-field TEM image, where the particles appear spherical with $D=7(1) \mathrm{nm}$, and HRTEM detail.

Simultaneously, an irreversibility appears for $T \lesssim 40 \mathrm{~K}$. These two findings disclose the magnetic reentrance. We have also applied different fields $(H \leqslant 10 \mathrm{kOe})$ to this ZFC-FC process and have plotted (see inset of Fig. 2) the $\delta T_{f}$ vs $H^{2 / 3}$ dependence, indicating that the glass temperature follows the de Almeida-Thouless line. This dependence was reported in bulk $\mathrm{Pb}\left(\mathrm{Fe}_{1 / 2} \mathrm{Nb}_{1 / 2}\right) \mathrm{O}_{3}$, where a coexistence of $\mathrm{AF}$ and $\mathrm{SG}$ order is put forward. ${ }^{15}$ In nanomagnets it has been found in $\gamma-\mathrm{Fe}_{2} \mathrm{O}_{3}$ (ferrimagnetic) and ascribed to the canting of the particle surface spins. ${ }^{23}$ The linear fit in the $1 / \chi$ vs $T$ Curie-Weiss plot provides $\theta_{P}=19(1) \mathrm{K}$, and a value of the paramagnetic moment $m_{\text {eff }}=9.83(5) \mu_{B}$, which compares well with the $\mathrm{Tb}^{3+}$ effective moment $\left(9.72 \mu_{B}\right)$. Here $\theta_{P}>$ 0 , in contrast to the bulk alloy value $\left(\theta_{P}=-7 \mathrm{~K}\right),{ }^{19}$ revealing

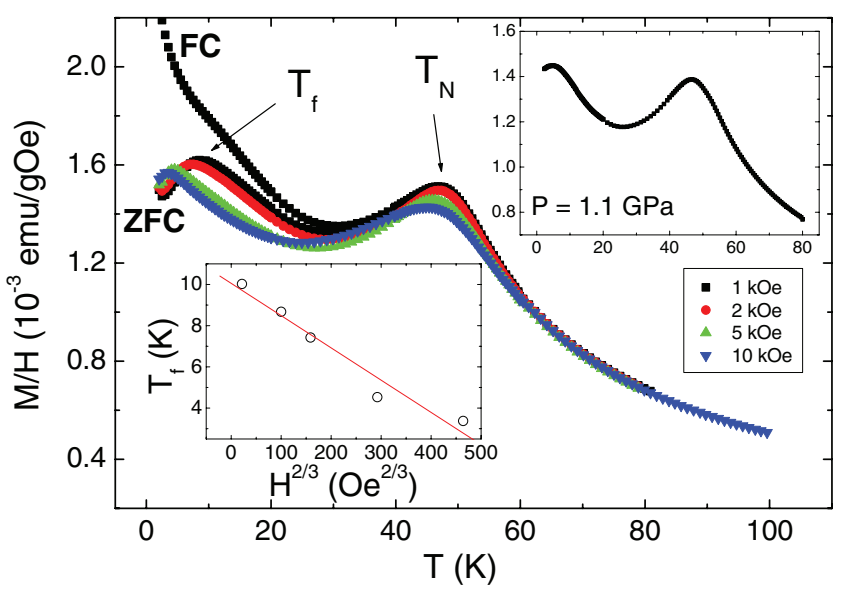

FIG. 2. (Color online) Thermomagnetic DC-susceptibility ZFC curves obtained with magnetic fields, $1 \mathrm{kOe} \leqslant H \leqslant 10 \mathrm{kOe}$, where the evolution from AF to SG state can be observed. A FC curve is also plotted at $H=1 \mathrm{kOe}$ to evidence the irreversibility. Inset (bottom): Linear $T_{f}$ variation vs $H^{2 / 3}$. Inset (top): ZFC DC susceptibility of the alloy at a pressure $P=1.1 \mathrm{GPa}$.

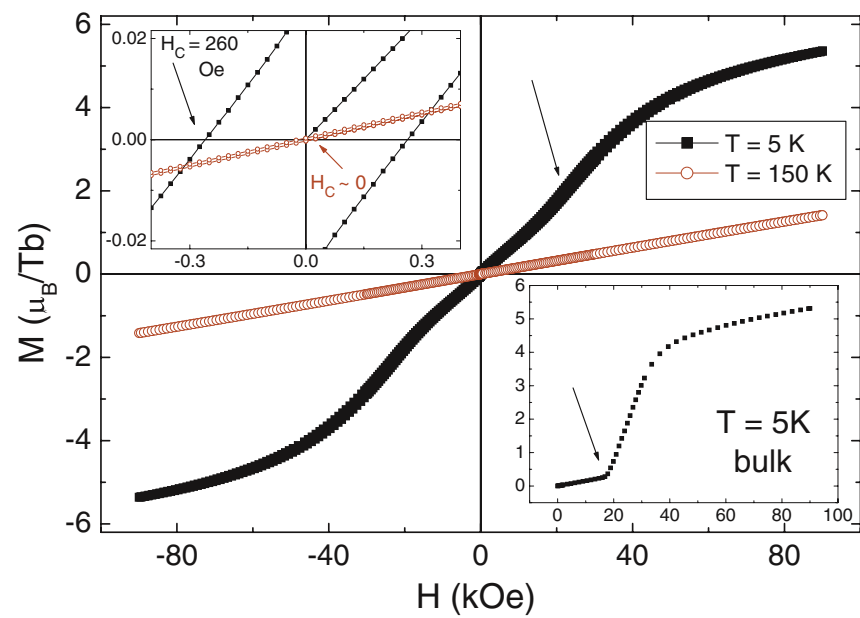

FIG. 3. (Color online) Magnetization curves of $\mathrm{TbCu}_{2}$ nanoparticles at $T=5 \mathrm{~K}$ (SG region) and $150 \mathrm{~K}$ (paramagnetic), up to $H=$ $90 \mathrm{kOe}$. Inset (left): Low-field coercivity in the SG state. Inset (right): $M_{\mathrm{DC}}(H)$ curves showing the metamagnetic transition of bulk alloy. Both bulk and $2 \mathrm{~h}$ alloys show the AF metamagnetic transitions, marked with arrows.

that the milling is triggering a weak FM coupling. The latter is necessary for the FM-AF frustration. ${ }^{2,4}$ The ordered magnetic arrangement is robust against hydrostatic pressure $(P)$ variations. In Fig. 2 (top inset) the $\mathrm{ZFC} M_{\mathrm{DC}}(T)$ curve under $P=1.1 \mathrm{GPa}$ is plotted. The $\mathrm{Cu}-\mathrm{Be}$ pressure cell is inside a SQUID magnetometer and the value of $P$ was extracted from the superconducting transition of $\mathrm{Sn}$. The effect of $P$ is negligible in $\Delta T_{N}(P) / T_{N}(P=0)=0.2 \%$ but $\Delta T_{f}(P) / T_{f}(P=0)=-15 \%$ is significant; the SG state is influenced by thermal and field history, ${ }^{2,4}$ and thus by external pressure. These transition temperatures are larger than in $\mathrm{Fe}_{0.55} \mathrm{Mg}_{0.45} \mathrm{Cl}_{2}\left(T_{N}=7.5 \mathrm{~K}, T_{f}=3 \mathrm{~K}\right),{ }^{11}$ and for the most recent $\mathrm{Pb}\left(\mathrm{Fe}_{1 / 2} \mathrm{Nb}_{1 / 2}\right) \mathrm{O}_{3}, T_{N}=7.5 \mathrm{~K}$ and $T_{f}=3 \mathrm{~K} .{ }^{15} \mathrm{In}$ $\mathrm{Co}_{2}(\mathrm{OH})\left(\mathrm{PO}_{4}\right), T_{N}=70 \mathrm{~K}, T_{f}=15 \mathrm{~K} .{ }^{13}$

In Fig. 3 , the $M_{\mathrm{DC}}(H)$ of the $\mathrm{TbCu}_{2}$ nanoparticles is examined up to $H=90 \mathrm{kOe}$. At $T=150 \mathrm{~K}$ the response is linear of PM character. Below $T_{f}$, a large signal is detected reaching $M_{\mathrm{DC}}(H=90 \mathrm{kOe})=5.35(5) \mu_{B} / \mathrm{Tb}$. The magnetization does not reach saturation, as occurs in $\approx 15 \mathrm{~nm} \mathrm{TbAl}_{2},{ }^{21}$ presumably due to the spin canting on the surface. ${ }^{16,23}$ Note that bulk $\mathrm{TbCu}_{2}$ is affected by a large anisotropy which complicates the determination of the saturation magnetization. ${ }^{24}$ The lack of saturation in the bulk alloy is confirmed in the bottom inset of Fig. 3. The extrapolation of the high-field magnetization to $H=0$ gives $M_{\text {nano }} / M_{\text {bulk }} \approx 80 \%$, hence a sign of the reduced contribution caused by moments at the surface. Another point is the existence of a kink around $H=20 \mathrm{kOe}$. This is a change of slope which is worth comparing to the $M_{\mathrm{DC}}(H)$ response in bulk $\mathrm{TbCu}_{2}$ (see bottom inset of Fig. 3). There, the kink is more pronounced and corresponds to the metamagnetic transition of AF. The outcome is that the AF is still present within the SG region. Regarding the presence of coercivity $\left(H_{C}=260 \mathrm{Oe}\right)$, which is also observed in SG systems, ${ }^{2,4,13}$ it should be noted that AF compounds should not display coercivity due to the absence of domains. The reentrant $\mathrm{Co}_{2}(\mathrm{OH})\left(\mathrm{PO}_{4}\right)$ shows $H_{C} \lesssim 50$ Oe. $^{13}$ 


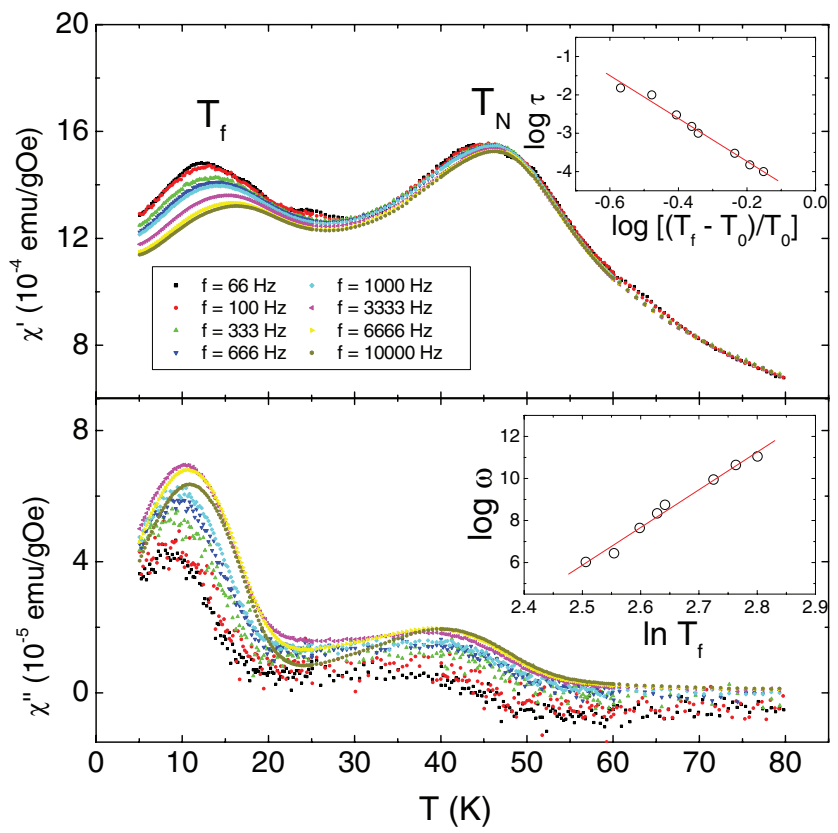

FIG. 4. (Color online) Real $\chi^{\prime}(T)$ (top) and complex $\chi^{\prime \prime}(T)$ (bottom) components of the AC susceptibility at several $f=\omega / 2 \pi$ ( $h=1 \mathrm{Oe})$. In $\chi^{\prime}(T)$, the Néel transition $\left(T_{N}\right)$ and the low-temperature freezing $\left(T_{f}\right)$ are detected. The latter clearly shifts to higher temperatures with increasing $f$. In the $\chi^{\prime \prime}(T)$, the AF transition is barely observed. Inset, bottom: Linear behavior of the $\delta$ shift of $T_{f}$. Inset, top: Line fit according to the critical slowing down of the relaxation times.

The spin dynamics was studied with an oscillating field $h=$ 1 Oe and the frequency was varied between $f=(\omega / 2 \pi)=$ $10 \mathrm{~Hz}$ and $10 \mathrm{kHz}$. In Fig. 4, the real $\left[\chi^{\prime}(T)\right]$ and complex $\left[\chi^{\prime \prime}(T)\right]$ components of the AC susceptibility are plotted for several frequencies. The shape of the $\chi^{\prime}(T)$ curve is similar to the ZFC $M_{\mathrm{DC}}(T)$. There is a main peak at $46.0(5) \mathrm{K}$ marking the $T_{N}$, practically frequency independent, and a smaller contribution, with a broad rounded peak which shifts up in temperature and decreases in magnitude with higher frequency, corresponding to $T_{f}(\omega)$. In the case of $\chi^{\prime \prime}(T)$, the Néel transition is nearly absent, marking the onset of antiferromagnetism, while the low $T_{f}$ peak is observable showing, as for $\chi^{\prime}(T)$, a $\omega$ shift. This shift is commonly quantified as $\delta=\Delta T_{f} / T_{f} \log _{10} \omega=0.057$ (3) (see bottom inset of Fig. 4). This value is larger than that of the individual spins in $\mathrm{Co}_{2}(\mathrm{OH})\left(\mathrm{PO}_{4}\right)(\delta=0.0024) .{ }^{13}$ If the magnetic relaxation of the particles were independent, a $\delta=0.1-0.2$ is expected. ${ }^{4,8}$ In ideal SGs the relaxation is faster and the values are much lower; ${ }^{2}$ for ferromagnets with reentrant state $\delta=0.01$ (in crystalline $\mathrm{FeAu}^{4}$ ) and $\delta=0.013(2)$ with data from amorphous $\left(\mathrm{Fe}_{0.20} \mathrm{Ni}_{0.80}\right)_{75} \mathrm{P}_{16} \mathrm{~B}_{6} \mathrm{Al}_{3} .{ }^{10}$ An intermediate metallic case is observed in super-SGs, as that of $\mathrm{Fe}_{24} \mathrm{Ag}_{76}$ with single-domain ferromagnetic Fe nanoparticles, where $\delta=0.010(1) .{ }^{9}$ Assuming that the spins participating in the freezing follow a critical slowing down, the measuring time $\tau_{m}$ : $\tau_{m}=\tau_{0}\left(T_{f} / T_{0}-1\right)^{-z v}$, where $z v$ is the dynamic exponent. Our analysis reveals a large $\tau_{0}=1.3(5) \times 10^{-5} \mathrm{~s}$, as that $\left(10^{-6} \mathrm{~s}\right)$ discussed in Fe-C $(4.7 \mathrm{~nm})$ nanoparticles, ${ }^{5} T_{0}=$ 9.6(4) $\mathrm{K}$, and $z v=5.7(1.1)$ (top inset of Fig. 4). The $z v$ value is close to reentrant FM and super-SG systems. ${ }^{8-10}$

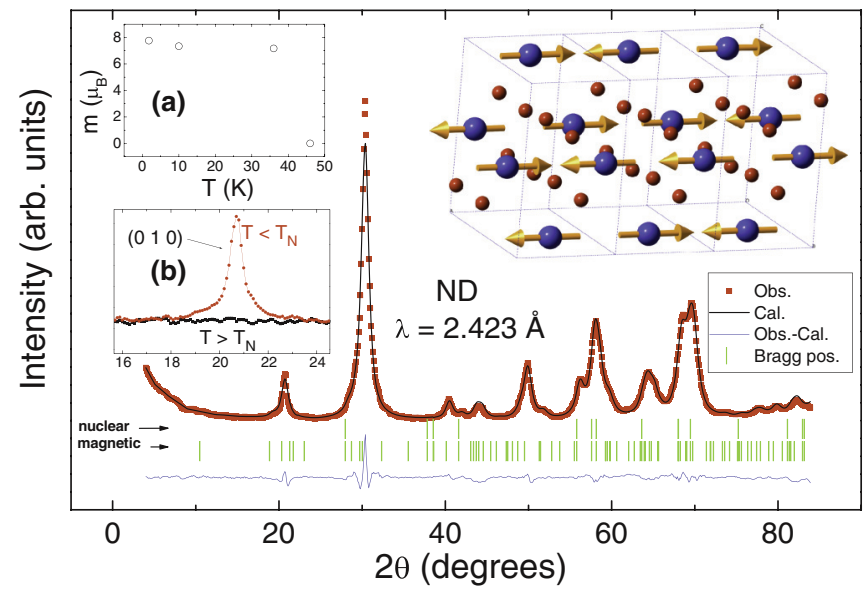

FIG. 5. (Color online) Neutron diffraction pattern at $T=1.8 \mathrm{~K}$ and its nuclear + magnetic Rietveld refinement. Right inset: The magnetic structure is antiferromagnetic (collinear) with large $\mathrm{Tb}$ atoms (blue) holding the magnetic moment (arrow) coinciding with that reported for bulk $\mathrm{TbCu}_{2}$ (Ref. 20). $\mathrm{Cu}$ atoms (red) are of smaller size. Inset (a): Thermal variation of the magnetic moment of $\mathrm{Tb}$ atoms. Inset (b): Uprise of first AF peak, below $T_{N}$.

So far the existence of macroscopic AF and SG states is clear. However a microscopic magnetic structure analysis is required; this has been carried out by neutron diffraction in Laboratoire Léon Brillouin (G4.1 instrument) with $\lambda=$ $2.423 \AA$ A. In Fig. 5, the neutron pattern at $T=1.8 \mathrm{~K}<T_{f}, T_{N}$, is shown together to a total Rietveld refinement. ${ }^{22}$ The lattice parameters are smaller: $a=4.3176(1) \AA, b=6.7842(9) \AA$, and $c=7.2990(7) \AA$, than the XRD ones at $300 \mathrm{~K}$. The size calculation yields similar $D_{\text {nuclear }}=9(1) \mathrm{nm}$ and $D_{\text {mag }}=$ 10 (1) $\mathrm{nm}$ values, establishing that the magnetic structure is a single domain in the particle. ${ }^{16,25}$ A rough estimation assuming the ratio of surface atoms $\left(N_{s}\right)$ with respect to those in the total volume $\left(N_{V}\right)$ through $N_{s} / N_{V}=(6 a / D) \approx 40 \%$, remarking the role of surface spins and supporting the surface moment reduction, although it only includes geometrical effects. A crucial result is that the magnetic structure (right inset of Fig. 5) is a commensurate AF with two propagation vectors $\vec{q}_{1}=(0,0,0)$ and $\vec{q}_{2}=(1 / 3,0,0)$, as in bulk. The ordered $T b^{3+}$ magnetic moment $m=7.76(2) \mu_{B}\left(R_{\text {mag }}=4.2 \%\right.$ and $R_{B}=1.1 \%$ ). In the temperature variation of the ordered magnetic moment [Fig. 5(a)], there is an expected increase below $T_{N}$ towards the magnetic ground state. The coherent diffraction across several lattice planes assures the long-range character of the AF interactions, with a mean correlation length $D_{\text {mag }}=10(1) \mathrm{nm}$ similar to the nanoparticle size. Hence the AF order survives in the frozen SG region [inset (b) of Fig. 5]. In fact the neutron magnetic intensity reduction $(\approx 78 \%)$ at $T=1.8 \mathrm{~K}$, considering the (neutron) bulk $m=8.8 \mu_{B},{ }^{20}$ also points to a disordered environment, affected by the decrease of surface coordination with respect to the atoms in the particles. ${ }^{16}$ Another revealing result is observed in Fig. 6. This is the existence of a clear $2 \theta<15^{\circ}$ low-angle scattering. The low-angle scattering increases with decreasing temperature evidencing magnetic correlations with an interparticle length scale. The $Q$ range is limited $\left(0.1 \AA^{-1}<Q<0.7 \AA^{-1}\right)$ but the variation of the integrated intensity confirms this result (left inset, Fig. 6). This is a sign of the existence of interparticle 


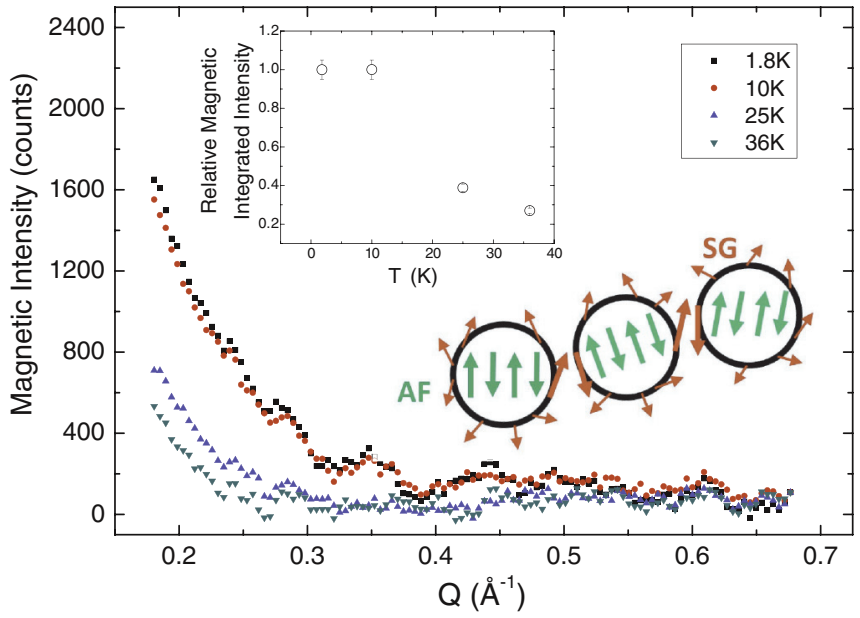

FIG. 6. (Color online) Low-angle magnetic contribution corresponding to the $0.1 \AA^{-1}<Q<0.7 \AA^{-1}$ range. There is a marked increase of curvature pointing to the existence of interparticle magnetic correlations. Left inset: Thermal variation of the integrated magnetic intensity for $Q<0.7 \AA^{-1}$. Right inset: Simplified sketch visualizing the spin nanostructure.

correlations. It is likely that reducing the $Q$ range would allow the precise determination of the coherence lengths involved. ${ }^{28,29}$ In short, there is an AF order within the percolating magnetic moments inside the particles (see sketch in Fig. 6).

In the few $\mathrm{AF}$ systems with a reentrant SG state, ${ }^{11-15}$ the combination of frustration and random bonds gives rise to the freezing. The Sherrington-Kirkpatrick model predicts that long-range ferromagnetism is always present but, for $T \leqslant T_{f}$, there exists a replica symmetry breaking resulting in the coexistence of FM and SG states. ${ }^{26}$ Such an explanation qualitatively supports the phase diagram in $\mathrm{Fe}_{0.55} \mathrm{Mg}_{0.45} \mathrm{Cl}_{2}$. ${ }^{11}$
For $\mathrm{Pb}\left(\mathrm{Fe}_{1 / 2} \mathrm{Nb}_{1 / 2}\right) \mathrm{O}_{3}$ both noncollinearity and clustering have been invoked. ${ }^{14,15} \mathrm{~A}$ very recent model has been proposed involving small clusters with AF order, showing $\chi(T)$ resembling those in Fig. 4, and predicting SG $\leftrightarrow$ PM transitions. ${ }^{27}$ However, here the particle size is much larger.

In our rare-earth alloy nanoparticulate system, the major AF coupling is held within the AF nanoparticles in a superantiferromagnetic arrangement and the uncompensated spins of both sublattices provide a source for randomness at the particle surface. As a result, a thin layer of random spins favors the SG behavior. It should be noted that although our experimental evidence is providing a single domain coherence length for each particle, it would not be surprising that such a coherence length could cover a few particles of the randomly placed particles, increasing the range of the AF interaction. The exact definition of such range caused by the RKKY interaction merits further work via small-angle neutron scattering. The spin scenario resembles that of amorphous $\mathrm{Fe}$-rich $\mathrm{FeZr}$, where magnetic clustering is settled in a metallic arrangement, ${ }^{6,29}$ which presents a reentrant macroscopic signature, and that of the supermagnetism appearing in nanomagnetic particles. ${ }^{7-9}$ Here, a fraction of spins forming the surface acts as a switch, enabling/disabling the coexistence, thus mimicking the variations of concentration in canonical and reentrant SGs. ${ }^{2,4,6}$

In conclusion, we have defined the Néel transition and the freezing temperature due to the spins at the surface of $\mathrm{TbCu}_{2}$ nanoparticles. The glassy transition does not hamper the AF state. The $\mathrm{PM} \leftrightarrow \mathrm{AF} \leftrightarrow \mathrm{SG}$ phases constitute a very attractive change of magnetic state in a metal. Consequently, the system should be considered as a superantiferromagnet with reentrant behavior assured by the nanoparticle surface.

This work was supported by a CICyT MAT2011-27573C04 grant. L.F.B. acknowledges pertinent comments by S. N Kaul (University of Hyderabad, India) and by S. G. Magalhães (Universidade Fluminense, Brazil).
${ }^{1}$ C. M. Hurd, Contemp. Phys. 23, 469 (1982).

${ }^{2}$ K. Binder and A. P. Young, Rev. Mod. Phys. 58, 801 (1986).

${ }^{3}$ E. C. Andrade and M. Vojta, Phys. Rev. Lett. 109, 147201 (2012).

${ }^{4}$ J. A. Mydosh, Spin Glasses: An Experimental Introduction (Taylor \& Francis, London, 1993).

${ }^{5}$ C. Djurberg, P. Svedlindh, P. Nordblad, M. F. Hansen, F. Bødker, and S. Mørup, Phys. Rev. Lett. 79, 5154 (1997).

${ }^{6}$ D. H. Ryan, J. M. D. Coey, E. Batalla, Z. Altounian, and J. O. Ström-Olsen, Phys. Rev. B 35, 8630 (1987); S. N. Kaul, V. Siruguri, and G. Chandra, ibid. 45, 12343 (1992).

${ }^{7}$ Steen Mørup, Morten Bo Madsen, Jørgen Franck, Jørgen Villadsen, and Christian J. W. Koch, J. Magn. Magn. Mater. 40, 163 (1983).

${ }^{8}$ S. Bedanta and W. Kleemann, J. Phys. D 42, 13001 (2009).

${ }^{9}$ J. Alonso, M. L. Fdez-Gubieda, J. M. Barandiarán, A. Svalov, L. Fernández Barquín, D. Alba Venero, and I. Orue, Phys. Rev. B 82, 054406 (2010).

${ }^{10}$ K. Jonason, J. Mattsson, and P. Nordblad, Phys. Rev. B 53, 6507 (1996).
${ }^{11}$ Po-zen Wong, S. von Molnar, T. T. M. Palstra, J. A. Mydosh, H. Yoshizawa, S. M. Shapiro, and A. Ito, Phys. Rev. Lett. 55, 2043 (1985); H. Yoshizawa, S. Mitsuda, H. Aruga, and A. Ito, ibid. 59, 2364 (1987).

${ }^{12}$ I. Mirebeau, C. Bellouard, M. Hennion, G. Jehanno, V. Caignaert, A. J. Dianoux, T. E. Phillips, and K. Moorjani, Physica C 184, 299 (1991).

${ }^{13}$ J. M. Rojo, J. L. Mesa, L. Lezama, J. L. Pizarro, M. I. Arriortua, J. Rodriguez Fernandez, G. E. Barberis, and T. Rojo, Phys. Rev. B 66, 094406 (2002).

${ }^{14}$ G. M. Rotaru, B. Roessli, A. Amato, S. N. Gvasaliya, C. Mudry, S. G. Lushnikov, and T. A. Shaplygina, Phys. Rev. B 79, 184430 (2009).

${ }^{15}$ W. Kleemann, V. V. Shvartsman, P. Borisov, and A. Kania, Phys. Rev. Lett. 105, 257202 (2010).

${ }^{16}$ S. Mørup, D. E. Madsen, C. Frandsen, C. R. H. Bahl, and M. F. Hansen, J. Phys.: Condens. Matter 19, 213202 (2007).

${ }^{17}$ L. Néel, Comptes Rendus Acad. Sci., Paris 253, 9 (1961). 
${ }^{18}$ D. P. Rojas, J. I. Espeso, L. Fernández Barquín, J. Rodríguez Fernández, and J. C. Gómez Sal, J. Magn. Magn. Mater. 310, e506 (2007).

${ }^{19}$ N. H. Luong and J. J. M. Franse, in Handbook of Magnetic Materials, edited by K. H. J. Buschow, Vol. 8 (Elsevier, Amsterdam, 1995), p. 415.

${ }^{20}$ V. Ma, Z. Smetana, B. Lebech, and E. Gratz, J. Magn. Magn. Mater. 54-57, 1357 (1986).

${ }^{21}$ D. P. Rojas, L. Fernández Barquín, J. Rodríguez Fernández, J. I. Espeso, and J. C. Gómez Sal, J. Phys.: Condens. Matter 19, 186214 (2007).

${ }^{22}$ J. Rodríguez-Carvajal, Physica B 192, 55 (1993).

${ }^{23}$ B. Martinez, X. Obradors, Ll. Balcells, A. Rouanet, and C. Monty, Phys. Rev. Lett. 80, 181 (1998).
${ }^{24}$ C. A. Poldy and E. Gratz, J. Magn. Magn. Mater. 8, 223 (1978).

${ }^{25}$ Franz Bødker, Mikkel F. Hansen, Christian Bender Koch, Kim Lefmann, and Steen Mørup, Phys. Rev. B 61, 6826 (2000).

${ }^{26}$ D. Sherrington and S. Kirkpatrick, Phys. Rev. Lett. 35, 1792 (1975).

${ }^{27}$ C. F. Silva, F. M. Zimmer, S. G. Magalhaes, and C. Lacroix, Phys. Rev. E 86, 051104 (2012)

${ }^{28}$ C. Bellouard, I. Mirebeau, and M. Hennion, Phys. Rev. B 53, 5570 (1996).

${ }^{29}$ R. García Calderón, L. Fernández Barquín, S. N. Kaul, J. C. Gómez Sal, Pedro Gorria, J. S. Pedersen, and R. K. Heenan, Phys. Rev. B 71, 134413 (2005). 